\title{
Occipital Nerve Stimulation for Headache Disorders
}

\author{
Koen Paemeleire* and Thorsten Bartsch ${ }^{\dagger}$ \\ *Department of Neurology, Ghent University Hospital, Ghent, B-9000 Belgium; ${ }^{\dagger}$ Department of Neurology, University Hospital \\ of Schleswig-Holstein, University of Kiel, Kiel, 24105 Germany
}

\begin{abstract}
Summary: Occipital nerve stimulation (ONS) was originally described in the treatment of occipital neuralgia. However, the spectrum of possible indications has expanded in recent years to include primary headache disorders, such as migraine and cluster headaches. Retrospective and some prospective studies have yielded encouraging results, and evidence from controlled clinical trials is emerging, offering hope for refractory headache patients. In this article we discuss the scientific rationale to use ONS to treat headache disorders, with emphasis on the trigeminocervical complex. ONS is far from a standardized technique at the moment and the recent literature on the topic is reviewed, both with respect to the procedure and its possible
\end{abstract}

complications. An important way to move forward in the scientific evaluation of ONS to treat refractory headache is the clinical phenotyping of patients to identify patients groups with the highest likelihood to respond to this modality of treatment. This requires multidisciplinary assessment of patients. The development of ONS as a new treatment for refractory headache offers an exciting prospect to treat our most disabled headache patients. Data from ongoing controlled trials will undoubtedly shed new light on some of the unresolved questions. Key words: Occipital nerve stimulation, refractory headache, cluster headache, migraine, hemicrania continua, neuropathic pain.

\section{REFRACTORY HEADACHES}

Despite a growing armamentarium of drugs, headache disorders can be refractory to medical treatment. The terms intractable headaches have often been used interchangeably with refractory headaches, although the latter is now preferred. ${ }^{1}$ Even though refractory headache is a well-recognized occurrence in clinical practice, little research has been performed on the topic and it is not defined in the International Classification of Headache Disorders, 2nd Edition (ICHD-II). ${ }^{2}$ A globally accepted definition does not exist today, but there have been a few attempts by the International Headache Society and the American Headache Society to develop operational definitions, such as those for migraine and cluster headaches. ${ }^{1,3}$ Patients with refractory headaches should have failed adequate trials of conventional drugs, because of unsatisfactory or lack of therapeutic effect, intolerable side effects, or contraindications to use. ${ }^{3}$ As the concept of refractory headaches is particularly developed for the purpose of controlled clinical trials that involve experi-

Address correspondence and reprint requests to: Koen Paemeleire, M.D., Ph.D., Department of Neurology, Ghent University Hospital, De Pintelaan 185, Ghent, B-9000 Belgium. E-mail: koen.paemeleire@ ugent.be. mental medication, invasive therapies, or implantable devices, it implies disability. ${ }^{3}$ Patients with refractory headaches lack a significant effect of drug treatment, but otherwise have very different conditions. Headache disorders are not part of a "continuum," but should be classified according to the ICHD-II, which is a hierarchical classification system (up to 4 digits) with diagnostic criteria, and it has three main categories: 1) primary headaches, 2) secondary headaches, and 3) cranial neuralgias. $^{2}$ There is a need for appropriate specific treatments for the various subtypes of refractory headaches, and studies are urgently required. Or to quote Dr. Jes Olesen: "No research can be done on a disease that is not defined ... it is difficult to define a disease on which no research has been done." 4 Developing the concept of refractory headaches is necessary for referral to empiric treatment, as well as inclusion in future clinical trials. Defining refractory headaches inevitably leads to the discussion of nonpharmacological options and may also create a basis for reimbursement of the medical costs of emerging interventional therapies. The conventional management options for medically intractable chronic headache syndromes are often limited and have been reviewed elsewhere., ${ }^{5,6}$ Various surgical procedures are offered to patients and neurostimulation procedures are of increasing interest. Peripheral nerve stimulation is a 
minimally invasive and reversible procedure, and is increasingly used in the treatment of certain forms of chronic neuropathic pain, where it is certainly preferred over nerve ablation procedures. Many targets for treating headache disorders and facial pain with neurostimulation have been described, including motor cortex, hypothalamus, thalamus, peri-aquaeductal grey, trigeminal tract, trigeminal nerve or ganglion, supra- and infra-orbital nerves, vagus nerve, and cervical spinal cord, but in recent years the main focus has been on stimulation of the occipital nerves in several headache disorders, including migraine and cluster headaches.

\section{OCCIPITAL NERVE STIMULATION: HISTORY}

Occipital nerve stimulation (ONS) was pioneered by Weiner and Reed, ${ }^{7}$ at the Departments of Neurosurgery and Anesthesiology, Presbyterian Hospital of Dallas, for the treatment of "C2-mediated headache" after they described a case series of 13 patients with intractable occipital neuralgia in $1999 .{ }^{8}$ However, the first use of ONS for headache was reported in 1977, including 6 patients, but no specific diagnoses were provided. ${ }^{9}$ Also in 1985 , ONS treatment for a patient with occipital neuropathy was described in a case series of patients with painful neuropathies treated with peripheral nerve stimulation. ${ }^{10}$ Initially, cuff electrodes twined around the nerve were used, but Weiner and Reed ${ }^{7}$ used subcutaneous cylindrical electrodes implanted at the occipitocervical junction. Beneficial effects of ONS were reported in more cases and case series, but the headache diagnosis sometimes remained as vague as: "Head pain that involved the $\mathrm{C} 2$ distribution with or without pain in other regions of the head" or "C2-mediated occipital headaches." ${ }^{, 11}$ Patients with primary headaches often report pain that involves not only the front of the head, innervated by the first (ophthalmic) division of the trigeminal nerve, but also the back of the head, mainly innervated by the greater occipital nerve that is a branch of the C2 spinal root. Eight patients of Weiner and Reed's ${ }^{7}$ series were further evaluated as part of a positron emission tomographic study. ${ }^{12}$ All eight patients were reclassified as chronic migraine patients according to the ICHD-II. In the past few years, the application of ONS has been widened to include a large number of primary and secondary headache disorders, such as migraine, chronic cluster headache, new daily persistent headache, hemicrania continua, chronic post-traumatic headache, chronic headache attributed to whiplash injury, cervicogenic headache, and occipital neuropathy. ${ }^{13-19}$ Identification of specific headache diagnoses that respond to ONS remains a challenge. ${ }^{13} \mathrm{Re}-$ cently, ONS has been evaluated in clinical trials in migraine and chronic cluster headaches. The Occipital Nerve Stimulation for the Treatment of Intractable Mi- graine (ONSTIM) trial in migraine has only been published in abstract form. ${ }^{18}$ There are two published trials in chronic cluster headache, one prospective and one retrospective, but these trials are uncontrolled, as they offer no comparison to sham. ${ }^{15,17}$

\section{ONS: Technique}

ONS is a minimal invasive procedure, with a stimulator implanted unilaterally or bilaterally at the level of the occipitocervical junction, such that stimulation causes slight paresthesia in the distribution of the occipital nerves after adjusting stimulation parameters, such as pulse width, frequency, and amplitude (FIG.1). Even though these are the basic characteristics of ONS, many technical variations exist, and there is a need for standardization. $^{20}$

Medial approach or lateral approach. The original technique described by Weiner and $\operatorname{Reed}^{7}$ used a lateral incision close to the mastoid process and a lead was advanced in the subcutaneous tissue toward the midline at the $\mathrm{C} 1$ level under fluoroscopy control. Later on, a medial approach was described with a midline incision. ${ }^{21}$ There are many arguments in favor of the medial approach. First, there is more subcutaneous fat at the midline, which allows surgeons to make a subcutaneous pocket large enough for adequate fixation of the lead, and leaving a loop to minimize lead dislocations. Second, this approach is favored especially with patients who

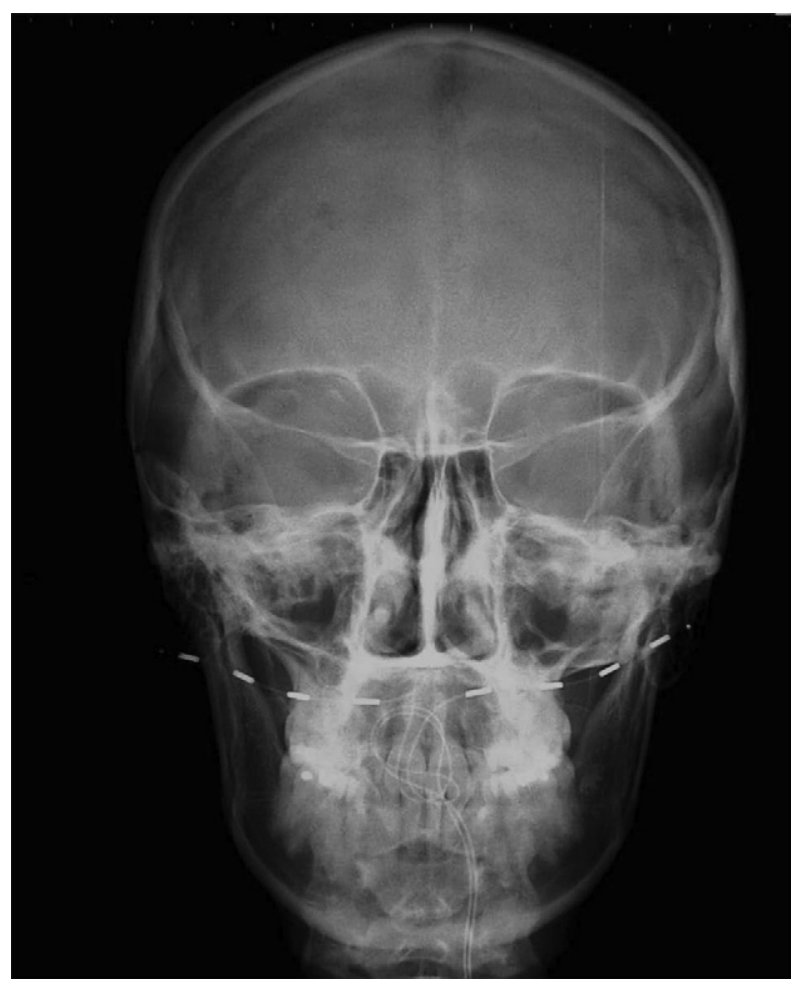

FIG. 1. Bilateral occipital nerve stimulators in place. (Courtesy of Dr. Jean-Pierre Van Buyten, Multidisciplinary Pain Centre, AZ Nikolaas, Sint-Niklaas, Belgium). 
wore glasses and complained of pain at the site of the incision with the lateral technique. Finally, the bilateral electrodes can be implanted with a single incision.

Types of devices. No specific electrodes have been developed yet for ONS. At present, ONS is typically performed with electrodes normally used for spinal cord stimulation. ONS electrodes exist as a paddle or cylindrical electrodes. One advantage of these electrodes is that electrodes for bilateral stimulation can be inserted through one midline incision. The paddle electrodes require more surgical dissection, but are associated with less scar tissue formation around the electrode, better stimulation field, and less change of migration. ${ }^{22}$ Silicone anchors and strain relief loops to reduce risk of migration are put in place. The ONS electrodes are connected to implantable pulse generators that can be nonrechargeable (i.e, with a lifespan of 2 to 5 years) or rechargeable. The implantable pulse generators can be implanted in the subclavicular, abdominal, or gluteal area. A recent development as an alternative to electrodes is the BION device. ${ }^{19,23}$ It is a rechargeable, telemetrically programmable, and current-controlled mini-neurostimulator. It has a cylindrical shape and is $27 \mathrm{~mm}$ in length and 3 $\mathrm{mm}$ in diameter. If bilateral ONS is required, a BION device should be implanted on the left and on the right.

Local or general anesthesia. Weiner and $\mathrm{Reed}^{7}$ described electrode placement under local anesthesia. Stimulation of the electrode during the procedure allowed the patient to indicate site of paresthesia, and therefore to verify correct electrode positioning relative to the occipital nerves. The procedure can also be performed under propofol sedation with a wake up during the procedure to check the area of paresthesia. However, experienced physicians now perform the procedure under general anesthesia with the patient in the prone position and the head in a horseshoe headrest. ${ }^{13,24}$ The authors ${ }^{7}$ argue that the added risk of general anesthesia is more than outweighed by the reduction in postoperative lead migration (i.e., the main adverse event). Indeed, electrode migration may occur in as many as $100 \%$ of individuals at 3 years follow-up, ${ }^{11}$ but this may be as low as $0 \%$ at 3 years after procedures using general anesthesia. ${ }^{13}$

Variability of the course of the greater occipital nerve. Important anatomic variability in the course of the occipital nerves exists, and in fact the classical descriptions do not seem to match recent data from cadaver studies. ${ }^{25}$ Placement of the occipital nerve stimulator above the nuchal line, which is higher than in the classical descriptions, may be associated with less muscle spasm while still providing paresthesia. ${ }^{26}$

\section{ONS: Mechanisms of action}

Anatomy of the nociceptive system of the head.

Three pairs of occipital nerves (i.e., the greater, lesser and third occipital nerves) provide sensory innervation of the back of the head on either side. Nociceptive fibers project to the upper cervical spinal dorsal horns that are continuous with the trigeminal nucleus caudalis, where nociceptive fibers of the trigeminal nerve synapse. Taken together, the upper cervical dorsal horns of $\mathrm{C} 1$ to $\mathrm{C} 3$ and the trigeminal nucleus caudalis form the trigeminocervical complex (TCC) (FIG. 2). From the TCC, nociceptive information is transmitted to higher centers in the brain. The TCC is a functional rather than anatomic entity, and physiological studies in animals have pointed at convergence of trigeminal and upper cervical nociceptive information, and thus a loss of spatial specificity at the level of the second order neurons of the TCC. ${ }^{27-29}$ The concept of TCC is furthermore supported by human experimental evidence. ${ }^{30,31}$ This functional continuum between occipital and trigeminal nociceptive input is important to understand as to how pain from a source in the neck can be referred to the trigeminal territory (in cervicogenic headaches), but it is equally important to note that primary headache disorders, such as migraine and cluster headache, characterized by activation of the trigeminovascular system, are often characterized by pain in the territory of the trigeminal and occipital nerves. The TCC itself is under the control of pain-modulatory structures, such as the periaquaeductal grey, the dorsolateral pon-

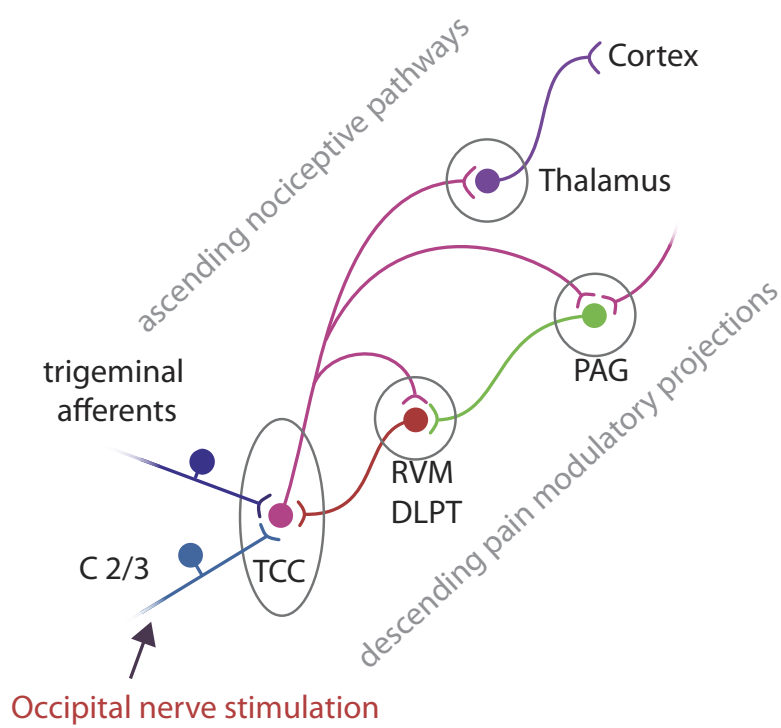

FIG. 2. Schematic drawing illustrating the functional anatomy of pain-modulatory pathways in the spinal cord and supraspinal structures. Nociceptive trigeminal fibers and C2 to C3 afferents synapse and converge in the trigeminal nucleus caudalis (TNC) and dorsal horns of C2 and C3. The dorsal horns C1 to C3 and the TNC form a functional continuum, the trigeminocervical complex (TCC), from which information is relayed to higher centers of the brain (e.g., the thalamus and cortex). Nociceptive and non-nociceptive information is relayed in the spinal dorsal horn where it is subject to segmental modulatory mechanisms either intrinsic or extrinsic from descending projections. The nociceptive input is transmitted to supraspinal relay sites, and is subject to inhibitory anti-nociceptive projections by pain-modulatory circuits in the brainstem. Pain processing on different levels may be modulated by neurostimulation of occipital nerves. DLPT = dorsolateral pontomesencephalic tegmentum; PAG = periaqueductal gray; RVM = rostral ventromedial medulla. 
tomesencephalic tegmentum and rostral ventromedial medulla oblongata. Together, these modulatory structures may generate both an anti-nociceptive or pro-nociceptive state of the TCC neurons.

Mechanim of action of ONS. ONS depolarizes the occipital nerves and anterograde impulses traverse in the sensory fibers toward the CNS. The beneficial effects of ONS in many different headache disorders suggest a nonspecific pain relief mechanism, although the mechanism of action may in fact be different depending on the condition. As shown by Magis et al. ${ }^{17}$ in patients suffering from chronic cluster headaches, ONS has neither a segmental nor a generalized analgesic effect.

Presently, the exact central and/or peripheral mechanisms, as well as the neurotransmitter systems involved are unknown. ONS may have central and peripheral effects that modulate nociception. It has been shown that electrical stimulation may change excitability of the peripheral nerve fibers themselves. ${ }^{32,33}$ Neurostimulation alters the conduction velocity and the amplitude of the A- $\alpha$, A- $\beta$, and especially A- $\delta$ fibers (the latter being involved in nociception) in isolated rat cutaneous nerve.

The understanding of pain-modulatory mechanisms in the spinal cord, as well as in the supraspinal structures, has been greatly advanced by the "gate-control theory" by Melzack and Wall. ${ }^{34}$ Although considerably extended and modified since 1965, this model in essence proposed that the transmission of pain in the spinal cord is modulated by excitatory and inhibitory influences. ${ }^{35}$ In accordance with the gate-control theory, an interplay of segmental spinal inhibiting effects and descending pain inhibitory pathways may also contribute to the analgesic effects of ONS. Given the loss of spatial specificity at the level of the trigeminocervical complex, electrical stimulation of the occipital nerve may have an anti-nociceptive effect in the territory of the trigeminal, as well as the occipital nerves. There is some animal evidence to support this notion, as stimulation of the greater occipital nerve in the rat reduces calcitonin gene-related peptide in the jugular blood, which is a biomarker of inhibition of the trigeminal system. ${ }^{36} \mathrm{~A}$ functional imaging study in chronic migraine patients supports the notion that ONS may influence supraspinal structures involved in central nociceptive trafficking, such as the dorsal rostral pons, the pulvinar nucleus of the thalamus and the anterior cingulate cortex. ${ }^{12}$ More details on the possible mechanism(s) of action, especially on the TCC, have been covered elsewhere. ${ }^{37}$ Interestingly, persistence of autonomic features has been described in one patient with hemicrania continua and one patient with chronic cluster headache after they obtained successful pain relief with ONS. ${ }^{38}$

\section{ONS: Efficacy}

The available data on ONS in primary headache disorders has recently been reviewed. ${ }^{39}$ Results on ONS in refractory chronic migraine, often in the context of medication overuse, have been encouraging, with at least $50 \%$ improvement in more than $80 \%$ of patients. Encouraging but less impressive results have emerged from the ONSTIM trial data, which are only available in abstract form. ${ }^{18}$ The ONSTIM trial is a prospective, multicenter, randomized, single blind, controlled feasibility study. The responder rate, defined as $50 \%$ drop in headache days per month or at least a three-point drop (on a 0 to 10 scale) in overall pain intensity from baseline at 3-month follow-up, was 39\% in refractory chronic migraine patients treated with ONS versus $8 \%$ in a control stimulation group and $0 \%$ in a medical management group. More randomized controlled trials, such as the Precision Implantable Stimulator for Migraine (PRISM) trial, are ongoing in chronic migraine. In a retrospective series including eight migraine patients, ongoing medication overuse was associated with a negative, long-term outcome. $^{13}$

In the chronic cluster headache, which is a devastating condition, results have been variable, and in the two largest case series (one prospective and one retrospective), at least $50 \%$ improvement was noted in approximately one third and two thirds of patients, respectively. ${ }^{16,17}$ In the prospective study, a delay of 2 months or more between implantation and significant clinical improvement was noted, which suggests that ONS acts via slow neuromodulatory processes in chronic cluster headaches. ${ }^{17}$ It seems reasonable to propose a trial of ONS in patients with drug-resistant chronic cluster headaches before considering hypothalamic deep-brain stimulation. $^{40}$

Hemicrania continua is characterized by an absolute response to indomethacin, but indomethacin may be contraindicated and its long-term use is often associated with side effects. More than $75 \%$ of hemicrania continua patients have a robust response to ONS with at least $50 \%$ improvement after a mean follow-up of approximately 1 year. ${ }^{19,39}$

Too few patients with paroxysmal hemicrania, shortlasting unilateral neuralgiform headache attacks, with conjunctival injection and tearing, and new daily persistent headache have been implanted to draw any conclusions. ${ }^{13,41}$

As for the secondary headache disorders and cranial neuralgias, data from case reports and case series are available in chronic post-traumatic headaches, chronic headache attributed to whiplash injury, cervicogenic headaches, occipital neuropathic pain (see ICHD-II 13.12) and occipital neuralgia. ${ }^{13,14}$ In a retrospective case series, eight patients with occipital neuropathic pain had an average overall percentage pain relief at long-term follow-up of $80 \% .^{13}$ All eight patients experienced pain relief within a week after the start of ONS treatment, even with some patients within the first 24 hours. Most, 
but not all, of these patients reported that the pain exacerbated very quickly (i.e., within 10 minutes to 1 hour) after switching the stimulator off.

\section{ONS: Issues}

There is a small, but growing body of evidence to support the efficacy of ONS in headache disorders. However, ONS should still be considered an experimental therapy, as many questions remain unanswered as of yet. There are no good predictors of efficacy; the response to occipital nerve block is not useful in predicting efficacy of ONS. ${ }^{42}$ The presence of an occipital component to the pain seems to have been the rationale in the past, although this is not required based on the physiology of the trigeminocervical complex. ${ }^{13}$ ONS has been used in many different situations, and identification of specific headache diagnoses, with a higher likelihood of responding to this modality of treatment, is required..$^{13}$ Multidisciplinary assessment of patients and clinical phenotyping of the headache syndrome, which may include an indomethacin test, is required in this respect. This requires harmonization of the classification systems of the International Association for the Study of Pain and the International Headache Society, which differ in many respects. Criteria for one condition may be different in the two classification systems, and occipital neuralgia is a typical example of this difference. Medication overuse was associated with a less favorable outcome in migraine patients in a retrospective series. ${ }^{13}$ Determining whether medication overuse is a predictor of negative outcome remains to be prospectively studied, but at the present time withdrawal from medication overuse is suggested prior to implantation as it may improve the patient's condition for a large part by itself.

One of the main areas of disagreement between pain physicians and neurologists is the relationship between headaches and the neck. Tenderness of the occipital nerves is seen as proof of origin of pain in the neck, but it is part of the phenotype of many headache disorders, such as migraines. ${ }^{43}$ Similarly, a response to occipital nerve block has been described in migraine and cluster headaches. ${ }^{44,45}$ Finally, even within the ICHD-II criteria, a lack of specificity to separate migraine from cervicogenic headaches has been suggested. ${ }^{46}$

As for the technique itself, the overall complication rate is low, however reinterventions are frequent and due to lead fracture, connector leakage, and need for battery replacement. ${ }^{13}$ The ideal positioning of the electrodes is still debated. Unpleasant local side effects may occur, such as muscle spasm, local discomfort, a shock-like sensation at the electrode site, as well as slight neck stiffness. Some have suggested an electrode placement higher at or above the nuchal line to avoid muscle spasms. ${ }^{26}$ Both traumatic and spontaneous electrode migration are frequently reported, with some series reporting as much as
$100 \%$ of cases at 3-year follow-ups. ${ }^{11}$ Lead pathway changes with movement have been modeled and implantable pulse generators in sites other than the buttock, including infraclavicular or low abdomen, may be associated with lower lead migration risk. ${ }^{47}$ Two cases of occipital lead tip erosion have been reported. ${ }^{48}$ There is no literature to show benefit of a trial period of ONS prior to implantation, although this is commonly performed (and may be required for reimbursement). The delay to clinical efficacy is variable between conditions, and may be as long as weeks or months in cluster headaches, ${ }^{16,17}$ but this can be experienced within a week in occipital neuropathy (see ICHD-II 13.12). ${ }^{13}$ A different mechanism of action of ONS in both conditions seems plausible but there is no physiological explanation yet. There are some arguments in favor of implanting bilateral stimulators in unilateral headache conditions, such as cluster headaches, as development of contralateral attacks has been described in cluster headache patients after unilateral implantation. ${ }^{17}$ The optimal stimulation parameters, such as pulse width, amplitude, and frequency, are now determined by trial and error, although a recently systematic study has begun with the BION device. $^{23}$ There are no data to correlate paresthesia maps with clinical outcomes. ${ }^{49}$

Suboccipital nerve stimulation is usually accompanied by local paresthesia, which makes the inclusion of a sham trial or a placebo arm difficult in ONS studies. Many data are gathered from retrospective series, which are associated with more sources of error. The results of the prospective ONSTIM trial in migraines are less impressive than what has been previously reported in retrospective series. ${ }^{18}$

\section{CONCLUSION}

ONS is a promising treatment, but far from proven. ${ }^{37}$ Some have suggested ONS is a useful tool in the treatment of chronic severe headaches with at least level IV (limited) evidence based on Agency for Healthcare Research and Quality (AHRQ) criteria. ${ }^{50}$ Patient satisfaction is generally high and ONS may have an effect even decades after onset of a headache disorder. ${ }^{13}$ The concept of refractory headache and its subtypes need to be further refined. A multidisciplinary approach is necessary to allow scientific evaluation of ONS on the basis of a specific headache diagnosis. This requires harmonization of existing classifications of the International Association for the Study of Pain and the International Headache Society. Withdrawal from medication overuse, especially in migraine patients, is necessary prior to implantation and may account for a large part of the improvement by itself. Prospective trials with sham control are eagerly awaited to assess the contribution of placebo effect, regression to the mean and spontaneous improvement in the observed effects. Preliminary ONSTIM trial 
results suggest that ONS is more effective than placebo or medical therapy, and that greater occipital nerve block may not predict response to ONS. ${ }^{18}$ Several clinical trials of ONS for chronic migraine are now in progress (NCT00286078; NCT00747812; NCT00200109).

Despite many unresolved questions, ONS is an exciting development with a huge potential to treat our most disabled and refractory headache patients, and is currently being offered on a compassionate basis as an off-label treatment.

\section{REFERENCES}

1. Schulman EA, Lake AE 3rd, Goadsby PJ, et al. Defining refractory migraine and refractory chronic migraine: proposed criteria from the Refractory Headache Special Interest Section of the American Headache Society. Headache 2008;48:778-782.

2. The International Classification of Headache Disorders, 2nd ed. Cephalalgia 2004;24(suppl 1):9-160.

3. Goadsby PJ, Schoenen J, Ferrari MD, Silberstein SD, Dodick D. Towards a definition of intractable headache for use in clinical practice and trials. Cephalalgia 2006;26:1168-1170.

4. Olesen J, Lipton RB. Headache classification update 2004. Curr Opin Neurol 2004;17:275-282.

5. Lake AE 3rd, Saper JR, Hamel RL. Comprehensive inpatient treatment of refractory chronic daily headache. Headache 2009;49: $555-562$.

6. van Kleef M, Lataster A, Narouze S, Mekhail N, Geurts JW, van Zundert J. Evidence-based interventional pain medicine according to clinical diagnoses. 2. Cluster headache. Pain Pract 2009;9:435442.

7. Weiner R, Reed K. Peripheral neurostimulation for control of intractable occipital neuralgia. Neuromodulation 1999;2:217-222.

8. Melvin EA Jr, Jordan FR, Weiner RL, Primm D. Using peripheral stimulation to reduce the pain of C2-mediated occipital headaches: a preliminary report. Pain Physician 2007;10:453-460.

9. Picaza JA, Hunter SE, Cannon BW. Pain suppression by peripheral nerve stimulation. Chronic effects of implanted devices. Appl Neurophysiol 1977;40:223-234.

10. Waisbrod H, Panhans C, Hansen D, Gerbershagen HU. Direct nerve stimulation for painful peripheral neuropathies. J Bone Joint Surg Br 1985;67:470-472.

11. Schwedt TJ, Dodick DW, Hentz J, Trentman TL, Zimmerman RS. Occipital nerve stimulation for chronic headache-long-term safety and efficacy. Cephalalgia 2007;27:153-157.

12. Matharu MS, Bartsch T, Ward N, Frackowiak RS, Weiner R, Goadsby PJ. Central neuromodulation in chronic migraine patients with suboccipital stimulators: a PET study. Brain 2004;127:220230.

13. Paemeleire K, Van Buyten JP, Van Buynder M, et al. Phenotype of patients responsive to occipital nerve stimulation for refractory head pain. Cephalalgia 2010 (in press).

14. Ghaemi K, Capelle HH, Kinfe TM, Krauss JK. Occipital nerve stimulation for refractory occipital pain after occipitocervical fusion: expanding indications. Stereotact Funct Neurosurg 2008;86: 391-393.

15. Burns B, Watkins L, Goadsby PJ. Treatment of medically intractable cluster headache by occipital nerve stimulation: long-term follow-up of eight patients. Lancet 2007;369:1099-1106.

16. Burns B, Watkins L, Goadsby PJ. Treatment of intractable chronic cluster headache by occipital nerve stimulation in 14 patients. Neurology 2009;72:341-345.

17. Magis D, Allena M, Bolla M, De Pasqua V, Remacle JM, Schoenen J. Occipital nerve stimulation for drug-resistant chronic cluster headache: a prospective pilot study. Lancet Neurol 2007; 6:314-321.

18. Goadsby PJ, Dodick DW, Saper JR, Silberstein S. Occipital nerve stimulation (ONS) for treatment of intractable chronic migraine (ONSTIM). Cephalalgia 2009;29:133.
19. Burns B, Watkins L, Goadsby PJ. Treatment of hemicrania continua by occipital nerve stimulation with a bion device: long-term follow-up of a crossover study. Lancet Neurol 2008;7:1001-1012.

20. Franzini A, Messina G, Leone M, Broggi G. Occipital nerve stimulation (ONS). Surgical technique and prevention of late electrode migration. Acta Neurochir (Wien) 2009;151:861-865.

21. Kapural L, Mekhail N, Hayek SM, Stanton-Hicks M, Malak O. Occipital nerve electrical stimulation via the midline approach and subcutaneous surgical leads for treatment of severe occipital neuralgia: a pilot study. Anesth Analg 2005;101:171-174.

22. Jones RL. Occipital nerve stimulation using a medtronic resume II electrode array. Pain Physician 2003;6:507-508.

23. Trentman TL, Rosenfeld DM, Vargas BB, Schwedt TJ, Zimmerman RS, Dodick DW. Greater occipital nerve stimulation via the Bion microstimulator: implantation technique and stimulation parameters. Clinical trial: NCT00205894. Pain Physician 2009;12: $621-628$.

24. Trentman TL, Zimmerman RS, Dodick DW, Dormer CL, Vargas BB. Occipital nerve stimulator placement under general anesthesia: initial experience with 5 cases and review of the literature. J Neurosurg Anesthesiol 2009 Oct 7; [Epub ahead of print].

25. Ducic I, Moriarty M, Al-Attar A. Anatomical variations of the occipital nerves: implications for the treatment of chronic headaches. Plast Reconstr Surg 2009;123:859-863.

26. Hayek SM, Jasper JF, Deer TR, Narouze SN. Occipital neurostimulation-induced muscle spasms: implications for lead placement. Pain Physician 2009;12:867-876.

27. Bartsch T, Goadsby PJ. Stimulation of the greater occipital nerve induces increased central excitability of dural afferent input. Brain 2002;125:1496-1509.

28. Bartsch T, Goadsby PJ. Increased responses in trigeminocervical nociceptive neurons to cervical input after stimulation of the dura mater. Brain 2003;126:1801-1813.

29. Goadsby PJ, Knight YE, Hoskin KL. Stimulation of the greater occipital nerve increases metabolic activity in the trigeminal nucleus caudalis and cervical dorsal horn of the cat. Pain 1997;73: 23-28.

30. Piovesan EJ, Kowacs PA, Tatsui CE, Lange MC, Ribas LC, Werneck LC. Referred pain after painful stimulation of the greater occipital nerve in humans: evidence of convergence of cervical afferences on trigeminal nuclei. Cephalalgia 2001;21:107-109.

31. Busch V, Jakob W, Juergens T, Schulte-Mattler W, Kaube H, May A. Functional connectivity between trigeminal and occipital nerves revealed by occipital nerve blockade and nociceptive blink reflexes. Cephalalgia 2006;26:50-55.

32. Ignelzi RJ, Nyquist JK. Direct effect of electrical stimulation on peripheral nerve evoked activity: implications in pain relief. J Neurosurg 1976;45:159-165.

33. Ignelzi RJ, Nyquist JK. Excitability changes in peripheral nerve fibers after repetitive electrical stimulation. Implications in pain modulation. J Neurosurg 1979;51:824-833.

34. Melzack R, Wall PD. Pain mechanisms: a new theory. Science 1965;150:971-979.

35. Dickenson AH. Gate control theory of pain stands the test of time. Br J Anaesth 2002;88:755-757.

36. Vincent MB, Ekman R, Edvinsson L, Sand T, Sjaastad O. Reduction of calcitonin gene-related peptide in jugular blood following electrical stimulation of rat greater occipital nerve. Cephalalgia 1992;12:275-279.

37. Goadsby PJ, Bartsch T, Dodick DW. Occipital nerve stimulation for headache: mechanisms and efficacy. Headache 2008;48:313318.

38. Schwedt TJ, Dodick DW, Trentman TL, Zimmerman RS. Occipital nerve stimulation for chronic cluster headache and hemicrania continua: pain relief and persistence of autonomic features. Cephalalgia 2006;26:1025-1027.

39. Bartsch T, Paemeleire K, Goadsby PJ. Neurostimulation approaches to primary headache disorders. Curr Opin Neurol 2009; 22:262-268.

40. Ambrosini A. Occipital nerve stimulation for intractable cluster headache. Lancet 2007;369:1063-1065.

41. Goadsby PJ. Neurostimulation in primary headache syndromes. Expert Rev Neurother 2007;7:1785-1789. 
42. Schwedt TJ, Dodick DW, Trentman TL, Zimmerman RS. Response to occipital nerve block is not useful in predicting efficacy of occipital nerve stimulation. Cephalalgia 2007;27:271274.

43. Bartsch T. Migraine and the neck: new insights from basic data. Curr Pain Headache Rep 2005;9:191-196.

44. Ambrosini A, Vandenheede M, Rossi P, et al. Suboccipital injection with a mixture of rapid- and long-acting steroids in cluster headache: a doubleblind placebo-controlled study. Pain 2005;118:92-96.

45. Afridi SK, Shields KG, Bhola R, Goadsby PJ. Greater occipital nerve injection in primary headache syndromes--prolonged effects from a single injection. Pain 2006;122:126-129.

46. Fishbain DA, Lewis J, Cole B, Cutler RB, Rosomoff RS, Rosomoff HL. Do the proposed cervicogenic headache diagnostic criteria dem- onstrate specificity in terms of separating cervicogenic headache from migraine? Curr Pain Headache Rep 2003;7:387-394.

47. Trentman TL, Mueller JT, Shah DM, Zimmerman RS, Noble BM. Occipital nerve stimulator lead pathway length changes with volunteer movement: an in vitro study. Pain Pract 2009 Oct 26; [Epub ahead of print]

48. Trentman TL, Dodick DW, Zimmerman RS, Birch BD. Percutaneous occipital stimulator lead tip erosion: report of 2 cases. Pain Physician 2008;11:253-256.

49. Terrence LT, Richard SZ, Nikesh S, Joseph GH, David WD Stimulation ranges, usage ranges, and paresthesia mapping during occipital nerve stimulation. Neuromodulation 2008;11:56-61.

50. Jasper JF, Hayek SM. Implanted occipital nerve stimulators. Pain Physician 2008;11:187-200. 